\title{
How the Saint Santin incoherent scatter system paved the way for a French involvement in EISCAT
}

\author{
P. Bauer ${ }^{1}$, A. Giraud ${ }^{2}$, W. Kofman ${ }^{3}$, M. Petit ${ }^{4}$, and P. Waldteufel ${ }^{5}$ \\ ${ }^{1}$ Bureau des longitudes, 23 Quai de Conti, 75006 Paris, France \\ ${ }^{2}$ Ecrivain scientifique, 5 rue de la clef, 75005 Paris, France \\ ${ }^{3}$ Institut de Planétologie et d'Astrophysique de Grenoble CNRS/UJF, 38000 Grenoble, France \\ ${ }^{4}$ Acdémie des sciences de Paris, 23 Quai de Conti, 75006 Paris, France \\ ${ }^{5}$ Institut Pierre-Simon Laplace, Université de Versailles Saint-Quentin-en-Yvelines, 78520 Guyancourt, France
}

Correspondence to: P. Bauer (pierre.bauer3@orange.fr)

Received: 29 May 2013 - Accepted: 20 August 2013 - Published: 13 September 2013

\begin{abstract}
This paper relates the development of a French incoherent scatter system which started its operations in 1965. This development took place several years after the initial implementation of such systems in the United States, in Peru and in the United Kingdom. The French system, owing to its bistatic configuration and the use of continuous waves, differed from the previous ones. These characteristics yielded signals of excellent spectral quality, unravelling the possibility of inferring physical parameters (Doppler shift, average ion mass) out of reach, at that time, of other systems. The possibility of making ion drift vector measurements led to extend the system into a quadristatic configuration. The multiple capabilities offered by the incoherent scatter technique, notably as concerns the thermodynamical properties of the ionosphere and of the thermosphere, led further the French community to a project of embarking an incoherent scatter radar on board a ship. Taking account of a project of a Scandinavian auroral zone radar and of the considerable interest of the study of auroral zone electrodynamics, the French community abandoned the idea of the ship and expressed an interest in joining the Scandinavian project in conjunction with Germany and the United Kingdom.
\end{abstract}

1

The scattering of electromagnetic waves by free electrons had been theoretically investigated at the beginning of the 20th century, first by Thomson (1906) in the case of a single electron and later by Fabry (1928) for a cloud of electrons. However one had to wait until 1958 when Gordon (1958) realized that powerful radars would be able to detect the faint waves scattered by the free electrons of the ionosphere. Bowles (1958) was the first to detect such signals with an experimental set-up at the University of Illinois and, very rapidly, impressive radar systems were implemented by the United States in Peru (1962) at Jicamarca $(300 \mathrm{~m} \times 300 \mathrm{~m}$ array of dipoles), in Massachusetts (1963) at Millstone Hill ( $70 \mathrm{~m}$ parabolic antenna) and in Puerto Rico (1964) at Arecibo (300 m spherical antenna, the largest worldwide!). France, for its share, initiated in 1961, during the "Gaullian era of big projects", the Saint Santin bistatic continuous wave system which started its operations in 1965. The Saint Santin system turned later onto a quadristatic configuration, which paved the way for a French involvement in EISCAT (European Incoherent Scatter).

\section{The French national centre for telecommunication studies (CNET) and the study of the ionosphere}

During the early 1960s, CNET, for the sake of its applications in the field of telecommunications, was deeply involved in fundamental studies of the propagation medium (atmosphere and ionosphere). As such, together with partners such as CNES (National centre for space studies), CNRS (National centre for scientific research), INAG (National institute for astronomy and geophysics), DRME (military research agency), CNET led major research endeavours such 


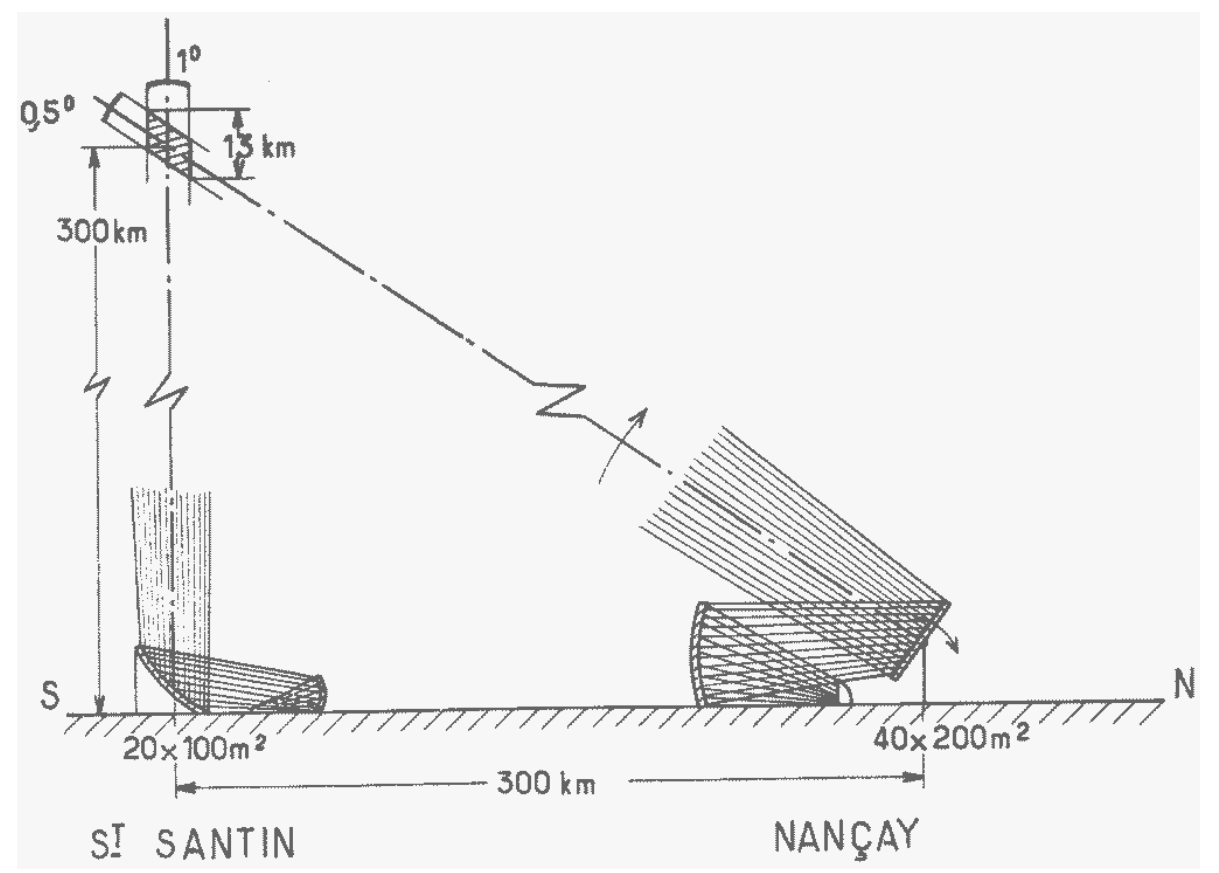

Figure 1. Set up of the Saint Santin incoherent scatter system. The transmitter located in Saint Santin illuminated a vertical column. The Nançay Radio telescope, located $300 \mathrm{~km}$ north of Saint Santin, scanned the vertical column in order to collect power scattered by the ionosphere.

as the development of FR1, a French satellite devoted to the study of the ionosphere and the magnetosphere, and the set up of ionospheric stations in Antarctica.

In 1961, Owen Storey, a scientist well known for his theoretical work on the whistlers (waves originating from lightnings and propagating along Earth's magnetic field lines), aware both of the pioneering incoherent scatter studies in the United States and of the decimetric radio telescope under construction in Nançay, suggested that this radio telescope should be used to collect the electromagnetic waves scattered by a vertical ionospheric column illuminated by a power transmitter properly located several hundreds of kilometres south of the radio telescope. In a matter of few days, the computations by a group from CNET, led by François du Castel, confirmed the soundness of the suggestion and gave rise to a proposal swiftly accepted by CNET and welcomed by the radio astronomy community which, under the leadership of Jean-François Denisse, had previously offered to provide up to $15 \%$ of the radio telescope observing time for geophysical research projects. The project, under the leadership of Michel Petit and through a close cooperation with the radio astronomy community, was ready to operate in 1965 at the time of completion of the radio telescope. It was in fact one of the first instruments to make use of the Nançay radio telescope. Far from being a project of the sole CNET group, it rapidly became a project of the whole French ionospheric community, notably in Toulouse and Grenoble.

\section{The Saint Santin incoherent scatter bistatic sounder}

As illustrated in Fig. 1, the Saint Santin incoherent scatter bistatic sounder (du Castel et al., 1966) operated with one transmitting antenna located in Saint Santin $300 \mathrm{~km}$ south of the Nançay radio telescope. The Saint Santin antenna illuminated a vertical column with a $140 \mathrm{~kW}$ continuous wave transmitter tuned at $935 \mathrm{MHz}$. The vertical column was scanned by the Nançay radio telescope. The main advantage of such a system was to avoid the convolution problems encountered with pulsed radar and therefore to yield signals of excellent spectral quality, unravelling the possibility of inferring physical parameters (Doppler shift, average ion mass) out of reach, at that time, of other systems.

\section{The non-linear regression analysis of the incoherent scatter spectrum}

A very important result of the Bowles experiment in 1958 (Bowles, 1958) was that while the return power appeared to be approximately of the order of magnitude predicted by Gordon (1958), the observed bandwidth was much narrower than predicted. This finding initiated considerable theoretical work on the plasma thermal fluctuations and the role of the ions (see Bauer, 1975, for a review). In turn, it was realized that the scattered signals would provide much more information on the ionospheric medium than initially thought. Quite 


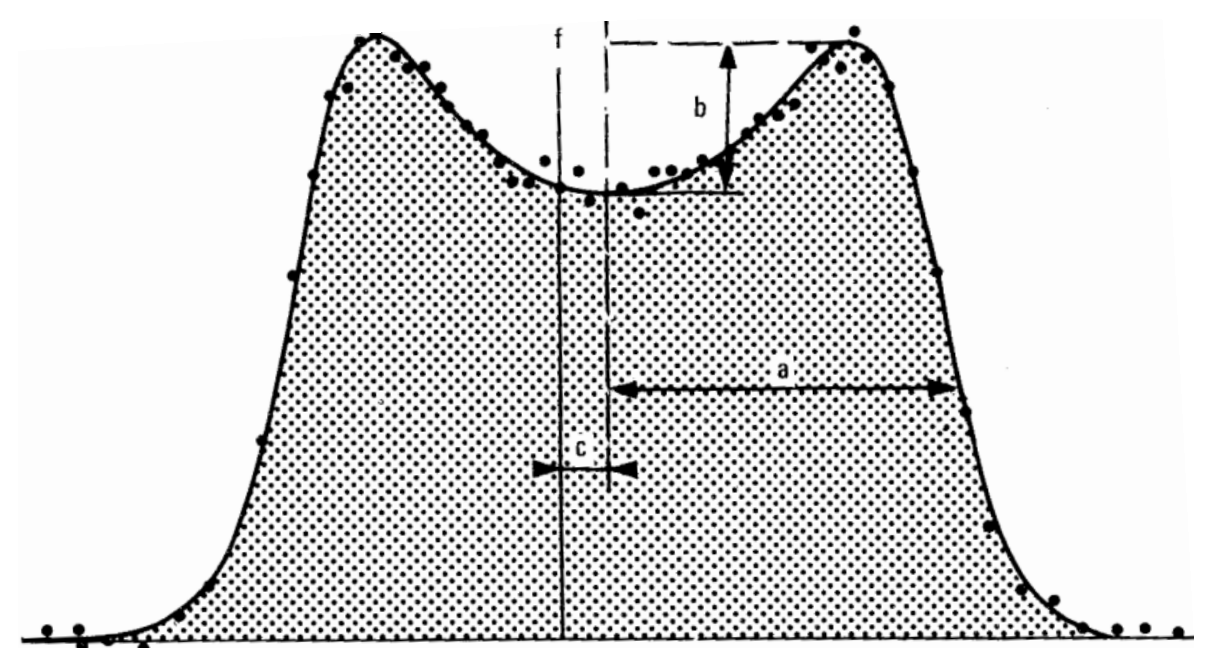

Figure 2. Best fit of an incoherent scatter spectrum to experimental data points. Parameters being fitted are the width "a", which depends on the electron temperature and the ion mass, the shoulder height " $b$ ", which is mainly a the ratio of electron to ion temperature, and the shift "c" from the transmitted frequency (here exaggerated for the benefit of clarity).

elaborated mathematical developments were to be made. Following Owen Storey's suggestions, Michel Petit developed in his thesis work (Petit, 1968) an optimal scheme to extract the physical parameters and the statistical errors attached to them, using the outstanding purity of the measured spectrum in $\mathrm{CW}$ (continuous wave) conditions.

His first step was to compute the probability of a particular observed signal throughout a time slot, taking into account the addition to the scattered signal of a much larger receiver (Gaussian) noise. The second step was to use a Bayesian approach to determine the physical medium parameters which would most likely induce the actually observed signal. The optimal treatment turned out to determine the physical parameters leading to a power spectrum best fitting the observed one during the time slot. This new approach developed by Petit was able to manage non-linearities and to provide, in addition to retrieved parameters, relevant estimates of theoretical standard deviations derived from each observation, as well as constraints on the bandwidth of the filters analysing the signal. The CNET team accessed to a recently developed, very efficient method to implement the retrieval. This method, called the Levenberg-Marquardt algorithm, was new in the 1960s. The perfect agreement of spectral data with theoretically modelled patterns (see Fig. 2), consistent with the signal to noise ratio, was a major incentive to the French community to go ahead with incoherent scatter. The flexibility of the processing later allowed taking into account additional parameters such as the global Doppler shift.

\section{Several significant achievements}

\subsection{Mapping of the thermospheric temperature}

Philippe Waldteufel and Philip McClure, making use of the thermodynamical coupling between the ionosphere and the thermosphere, produced a first preliminary global map of the thermospheric temperature based on the measurements at Jicamarca $\left(11^{\circ} \mathrm{S}\right)$ and Saint Santin $\left(44^{\circ} \mathrm{N}\right)$ (Waldteufel and McClure, 1969). For this purpose a seasonal symmetry of the two hemispheres was assumed. While up to that time the thermospheric temperature was simply used as a parameter in order to model empirically the density profiles, it was found indeed that this parameter did not differ too much from the actual physical temperature. Later on, in 1975, by combining data from all the incoherent scatter observatories and satellites data a more accurate global modelling of the thermosphere became available. It showed that the original work of Waldteufel and McClure was not too far from reality.

\subsection{Ionospheric dynamics}

Michel Petit, noticing that in many cases the best fits were systematically smaller than the actual experimental data on one side of the power spectrum and larger on the other side, realized that the small discrepancy would be eliminated by a small frequency shift of the whole spectrum, i.e. that the Doppler shift induced by the plasma motion along the line of sight, as small as few $\mathrm{Hz}$ for a $10 \mathrm{kHz}$ wide spectrum, was easily detectable. This showed that having very stable absolute frequency references for the transmitter and the receiver allowed making very precise $\left(1 \mathrm{~ms}^{-1}\right)$ velocity measurements (Petit, 1968). This finding opened a considerable avenue to the study of ionospheric dynamics (including electric field induced motions) and paved the way to the design 


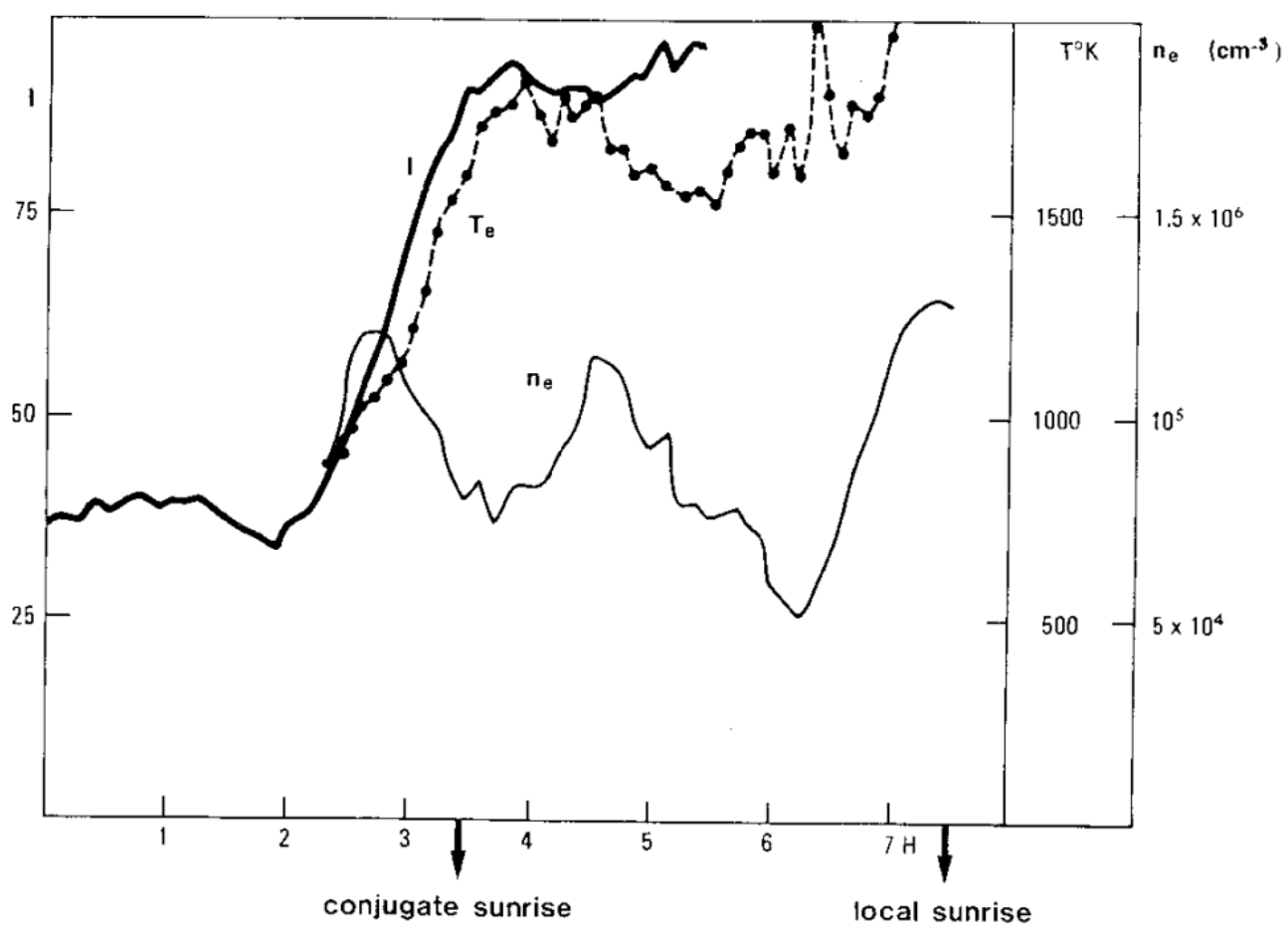

Figure 3. Simultaneous measurements of the oxygen line (I in Raleigh), of the electron temperature $\left(T_{\mathrm{e}}\right.$ in $\left.{ }^{\circ} \mathrm{K}\right)$ and of the electron concentration $\left(n_{\mathrm{e}}\right.$ in $\left.\mathrm{cm}^{-3}\right)$ (Duboin et al., 1968).

of multistatic facilities, able to measure the three components of the ions' mean velocity.

\subsection{Observing the Saint Santin magnetically conjugated sunrise}

A spectacular effect due to photoelectrons that escape into the magnetosphere used to be observed during winter nights at time of sunrise at the magnetically conjugate ionosphere. While this phenomenon was initially detected by Carlson at Arecibo (Carlson Jr., 1966), its occurrence at Saint Santin was particularly amplified due on one hand to the higher latitude of Saint Santin and on the other hand the eastward longitude of its conjugate point. Photoelectrons being created in the conjugate ionosphere, approximately one hour before sunrise at the ground level, would travel along the magnetic field line, heat the local electrons, thus raising the electron temperature, and excite oxygen atoms, triggering an increase of the intensity of the $630 \mathrm{~nm}$ oxygen line above the level resulting from recombination processes. This is illustrated in Fig. 3 (Duboin et al., 1968). The conjugate photoelectron flux progressively increases for about one hour, before reaching its maximum value (for a small optical depth in the conjugate ionosphere). This increase is accompanied by progressive electron temperature and oxygen line intensity increases.

\section{Evolving towards a quadristatic system}

The capability of detecting Doppler shifts with an excellent accuracy led very rapidly to the concept of three-dimensional (3-D) wind measurements. In order to do so it was necessary to add two receiving stations $100 \mathrm{~km}$ east and west of Saint Santin. The proposal for such an extension was submitted in 1968 (Waldteufel, 1967) to INAG, which accepted to provide its funding. The extended incoherent scatter sounder started to operate in 1973. This gave a strong impulse for the study of the electrodynamical properties of the ionosphere.

\section{The project of an incoherent scatter radar on board a ship}

By the end of the 1960s the incoherent scatter devices had demonstrated their powerful ability to shed light on most of the fields of ionospheric and thermospheric physics. They suffered however a weakness, since it was not possible to move such huge devices to the very places where they were often needed. In this context the idea to embark a radar on a ship was born. The suggested configuration was a monostatic radar, with pointing ability in order to keep the 3-D wind measurement capability demonstrated at Saint Santin (Waldteufel, 1968). 


\section{The choice between the Scandinavian and the French projects}

A Scandinavian auroral zone project (see, for example, Hultqvist, 2011) and the French ship project where both supported by the 1969 URSI General Assembly, several factors would concur to the merging of the two projects. While many teams across the world expressed interest in the ship borne incoherent scatter concept, a major difficulty arose about the cost (both investment and running), heavily increased by the ship's logistics. Simultaneously, the possibility of measuring ion drift vectors initially inferred by the "Saint Santin" system revealed to be one of the main drivers for future incoherent scatter projects, in particular for the study of the ionosphere at high geomagnetic latitudes and its coupling to the magnetosphere. Finally, the idea of building another great European project after those elaborated in nuclear physics and in astronomy was a strong driver. All these elements pushed for the Scandinavian project. Based on a proposal by Owen Storey, CNRS, led by its deputy director Pierre Creyssel, expressed an interest in joining the Scandinavian project in conjunction with Germany and the United Kingdom.

\section{The wide band correlator for plasma line measurements: from Chatanika to Tromsø}

In the early 1970s the French group decided to build a fast correlator in order to measure the plasma line part of the incoherent spectra. The problem was to cover a wide band instantaneously because the plasma line can drift rapidly between a few hundred of $\mathrm{kHz}$ up to $10 \mathrm{MHz}$, especially in the auroral zone.

The study of the correlator started in 1973 in LETI Grenoble (Chabert et al., 1974), and the correlator was built in 1975. In the 1970s the fast electronics was not yet well developed and to build a fast correlator with a large number of quantification bits was not possible. For the integrated circuits technology the speed of calculations was a major limitation. The speed of calculation imposed a limit to the sampling rate and the number of bits in ADC (analog to digital convertor). The adopted solution was to use 2 bits + sign (7 levels of quantification). The uniformly distributed noise was added to the signal, with the amplitude of one quantization level, to linearize the quantification. This permitted one to have an unbiased estimator of the correlation function. With these constraints it was possible to build the correlator working with the sampling rate up to $15 \mathrm{MHz}$ and calculating 4 correlation functions (gates) of 256 points. This was possible on separated time intervals. The results were accumulated and stored in 4 memories of 256 words of 32 bits each. The correlator worked at the limit of electronics speed and the power consumption was extremely huge $(4 \mathrm{~kW})$. This power consumption was in fact so large that, in Alaska operating with an outside temperature of $-40^{\circ} \mathrm{C}$, all doors had to be left open in order to keep the inside temperature at a reasonable level.

The correlator was used extensively up to 1983 and then, with the fast development of technology, begun to be obsolete.

The first measurements using the correlator were made at the French quadristatic incoherent scatter facility (19751980) (Kofman et al., 1981). It was then demonstrated that the plasma lines could be used to determine the electron temperature in the $\mathrm{F}$ region of the ionosphere. The correlator was then transported to the Chatanika incoherent scatter radar (1978-1979) (Kofman and Wickwar, 1980). The main scientific result was the detection of the Doppler shift between the up- and down-shifted plasma lines. These shifts were in turn used to estimate the Birkeland current carried by ambient electrons (Kofman and Wickwar, 1980). In addition, the first measurements in the auroral zone of photoelectron-excited plasma lines in the ionosphere were also obtained. Finally, the correlator was operated at the EISCAT Troms $\varnothing$ facility (1981-1983) (Kofman et al., 1982; Folkestad et al., 1983; Hagfors et al., 1983). EISCAT measurements were used to calibrate electron density measurements and to obtain initial observations of heater-induced plasma line enhancements.

All the correlator campaigns resulted in a very fruitful cooperation with the Chatanika (SRI) and EISCAT teams.

\section{Concluding remarks}

The contributors of this paper, who have participated enthusiastically in the fascinating adventure of the "incoherent scatter sounding of the ionosphere" have all, except one (Wlodek Kofman), gone a long path away from their initial involvement in EISCAT; they have turned to meteorology, climate studies, telecommunications, space activities and others. They all keep, however, of the setting up of EISCAT, the feeling of a beautiful European endeavour. They remember in particular the swift and forceful brainstorming of a consortium of experts of the different EISCAT countries, led by Tor Hagfors, in order to recover "a loss of know how" in the design of the UHF klystrons, a long-standing problem which delayed the initial operations of the UHF system by many years; a good example of European cooperation. Wlodek Kofman, who continued to be a very active EISCAT scientist, is now trying to grasp a new "Ice cat" with the European Space Agency mission ROSETTA heading towards the icy nucleus of a comet. He is the prime investigator of a radar system aiming to determine the tomography of the nucleus at around 2015.

Edited by: A. Brekke

Reviewed by: B. Hultqvist and O. Holt 


\section{References}

Bauer, P.: Theory of waves incoherently scattered, Phil. Trans. R. Soc. Lond. A., 280, 167-191, 1975.

Bowles, K. L.: Observation of vertical incidence scatter from the ionosphere at $41 \mathrm{Mc} / \mathrm{sec}$, Phys. Rev. Lett., 1, 454-455, 1958.

Carlson Jr., H. C.: Ionospheric heating by magnetic conjugate-point photoelectrons, J. Geophys. Res., 71, 195-199, 1966.

Chabert, B., Dutang, P., and Sanchez, G.: Correlateur multibit a large bande (16 MHz), Note Tech. LETI/MCTE 1045 CEACENLab. d'Electron, et de Technol. de l'Informatique, Grenoble, 1974.

Duboin, M. L., Lejeune, G., Petit, M., and Weill, G.: Excitation of oxygen lines and calculations of the conjugate predawn effects, J. Atmospheric Terrest. Phys., 27, 489-495, 1968.

du Castel, F., Carru, H., Petit, M., and Waldteufel, P.: Le sondeur ionosphérique à diffusion du CNET, Onde électrique, 468, 281284, 1966.

Fabry, C.: Remarques sur la diffusion de la lumière et des ondes hertziennes par les electrons libres, C. r. hebd. Séance Acad. Sci. Paris, 187, 771-781, 1928.

Folkestad, K., Hagfors, T., and Westerlund, S.: EISCAT: An updated description of technical characteristics and operational capabilities, Radio Sci., 18, 867-879, 1983.

Gordon, W. E.: Incoherent scattering of radio waves by free electrons with applications to space exploration by radar, Proc. I. R. E., 46, 1824-1829, 1958.

Hagfors, T., Kofman, W., Kopka, H., Stubbe, P., and Aijanen, T.: Observations of enhanced plasma lines by EISCAT during heating experiment, Radio Sci., 18, 861-866, 1983.
Hultqvist, B.: History of EISCAT - Part 1: On the early history of EISCAT with special reference to the Swedish part of it, Hist. Geo Space. Sci., 2, 115-121, doi:10.5194/hgss-2-115-2011, 2011.

Kofman, W. and Wickwar, V.: Plasma Line measurements at Chatanika with high-speed correlator and filter bank, J. Geophys. Res., 85, 2998-3012, 1980.

Kofman, W., Lejeune, G., Bauer, P., and Hagfors, T.: Electron Temperature Measurements by the Plasma Line Technique at the French Incoherent Scatter Radar, J. Geophys. Res., 86, 67956801, 1981.

Kofman, W., Lejeune, G., Bauer, P., Hagfors, T., and Folkestad, K.: EISCAT First Plasma Line Experiment, Ann. Geophys., T38, 461-466, 1982.

Petit, M.: Mesures de températures, de densité électronique et de composition ionique, dans l'ionosphère par diffusion de Thomson, Étude du déséquilibre thermodynamique dans l'ionosphère diurne, Ann. Geophys., 24, 1-38, 1968, http://www.ann-geophys.net/24/1/1968/.

Thomson, J. J.: Conduction of electricity through gases, 321, London, Cambridge University Press, 1906.

Waldteufel, P.: Projet de développement du sondeur à diffusion français, Note CNET/RSR/1225, 1967.

Waldteufel, P.: Some preliminary considerations for an incoherent scatter radar embarked on a ship, Note CNET/RSR, 1968.

Waldteufel, P. and McCLure, J. P.: Preliminary comparisons of middle and low latitude Thomson scatter data, Ann. Geophys., 25, 785-793, 1969,

http://www.ann-geophys.net/25/785/1969/. 


\section{My recollections of EISCAT by Alain Giraud}

I was involved with the EISCAT project in 1974-1975, rather briefly, at the request of François du Castel, who was one of my bosses in the CNET-CNRS lab where I had worked for the past ten years on ionospheric research, implementing radar and rocket soundings of the upper atmosphere.

CNRS is the French government's main research institution, overseeing and financing scientific programs in every academic field. CNET on the other hand was something like the French equivalent of the Bell Labs, mostly involved in telecommunications developments. For CNRS, EISCAT was a venture in competition with IRAM, a projected millimetre wave radio astronomy facility in the south of France. For CNET, EISCAT was very marginal, especially after the presidential election in 1974, when updating the national telephone network (then in a sorry state after decades of neglect) became a political priority.

Du Castel had negotiated the early phases of the project, but was called on to become scientific director of CNET, with wide ranging responsibilities, and asked me to take over with EISCAT. A couple of years later he was to be appointed as one of the heads of CNET, and I was to succeed him at the scientific direction. In the meantime, while completing various other assignments, I spent about half of my working time preparing and attending EISCAT meetings.

It was an exciting experience because the project was nearing maturity and that was the time when most decisions were actually taken.

We were lucky in having at CNRS a very smart and energetic top administrator, Pierre Creyssel, who considered that EISCAT as well as IRAM were both worthwhile ventures, and rather than choosing one over the other was intent on financing both. He therefore kept pushing for all of us to reduce costs, so that they would fit into the overall available budgetary envelope. They did.

As I remember it, my contribution was dictated by this goal, and materialized in organizational and technical savings, as Thor Hagfors was kind enough to acknowledge in a warm letter he wrote me when I quit in 1975 (his letter is the only archive I have kept of this period of my life).
On the organizational level the idea was to establish a wall between funding and spending. I advocated pooling resources into a common fund, and allocating spending to the best priced offers in international calls for bid, irrespective of national preferences. That was in obvious opposition to the then prevalent principle of juste retour, stipulating that each country should get back in orders an amount equivalent to its investment.

On the technical level, that meant departing from the equally prevalent principle that each country should design and provide a fully operational piece of equipment. Rather, the management of the project should take full responsibility to optimize designing the system and allocating the parts to best offer providers, irrespective of nationality; that resulted in significant savings, not only from increased competition, but in system design - like for instance having a common power system for the UHF and VHF transmitters instead of separate full-fledged transmitters.

But my best memories from these two years, all in all quite successful, have little to do with science or management. The EISCAT meetings, although involving lot of work and stress, were also a kaleidoscope of discoveries and hospitalities - countries, cities, cultures, people. I am sure our British, Scandinavian and German colleagues share or have shared the same fond recollection of our gatherings that I do: the flowering eloquence of the Monpazier mayor in Périgord over foie gras and Cahors Cabernet, the post-sauna barbecued sausage and beer in the snow at Södankyla, the great fish restaurants and Chardonay in Troms $\varnothing$, hotel Ferrum in Kiruna, not to mention Hamburg, Munich or, what else, London!

As far as I am concerned, that is when I became a European, a forever europtimist in fact. 